\title{
Vestibulocochlear Symptoms Caused by Vertebrobasilar Dolichoectasia
}

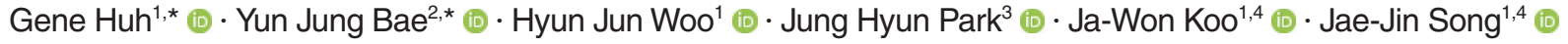 \\ Departments of ${ }^{l}$ Otorhinolaryngology-Head and Neck Surgery and ${ }^{2}$ Radiology, Seoul National University Bundang Hospital, Seongnam; \\ ${ }^{3}$ Department of Radiology, Ajou University Hospital, Ajou University School of Medicine, Suwon; ${ }^{4}$ Dizziness Center, Seoul National University \\ Bundang Hospital, Seongnam, Korea
}

Objectives. Vertebrobasilar dolichoectasia (VBD), an elongation and distension of vertebrobasilar artery, may present with cranial nerve symptoms due to nerve root compression. The objectives of this study are to summarize vestibulocochlear manifestations in subjects with VBD through a case series and to discuss the needs of thorough oto-neurotologic evaluation in VBD subjects before selecting treatment modalities.

Methods. Four VBD subjects with vestibulocochlear manifestations were reviewed retrospectively. VBD was confirmed by either brain or internal auditory canal magnetic resonance imaging (MRI) and magnetic resonance angiography (MRA). Patient information, medical history, MRI/MRA findings, and audiometry or vestibular function tests were reviewed according to patient's specific symptom.

Results. Of the four subjects, three presented with ipsilesional sensorineural hearing loss (SNHL), three with paroxysmal recurrent vertigo, and two with typewriter tinnitus. The MRI/MRA of the four subjects revealed unilateral VBD with neurovascular compression of cisternal segment or the brainstem causing displacement, angulation, or deformity of the cranial nerve VII or VIII that corresponded to the symptoms.

Conclusion. Vestibulocochlear symptoms such as SNHL, recurrent paroxysmal vertigo, or typewriter tinnitus can be precipitated from a neurovascular compression of the vestibulocochlear nerve by VBD. Because proper medical or surgical treatments may stop the disease progression or improve audio-vestibular symptoms in subjects with VBD, a high index of suspicion and meticulous radiologic evaluation are needed when vestibulocochlear symptoms are not otherwise explainable, and if VBD is confirmed to cause audiovestibular manifestation, a thorough oto-neurotologic evaluation should be performed before initial treatment.

Keywords. Sensorineural Hearing Loss; Hemifacial Spasm; Microvascular Decompression

\section{INTRODUCTION}

Vertebrobasilar dolichoectasia (VBD) [1] is a rare arteriopathy lacking a clear etiology. The principal VBD pathophysiology may

\footnotetext{
- Received May 23, 2019

Revised July 15, 2019

Accepted August 4, 2019

- Corresponding author: Jae-Jin Song

Department of Otorhinolaryngology-Head and Neck Surgery, Seoul

National University Bundang Hospital, 82 Gumi-ro 173beon-gil,

Bundang-gu, Seongnam 13620, Korea

Tel: +82-31-787-7408, Fax: +82-31-787-4057

E-mail: jjsong96@snubh.org

*These authors contributed equally to this work.
}

reflect aberrant vascular remodeling and degradation of the arterial wall caused by increased blood pressure [2]. The prevalence of VBD ranges from $0.2 \%$ to $4.4 \%$ [3], but precise data on general populations are lacking. Old age, the male sex, hypertension, smoking, and a history of myocardial infarction have been suggested to be risk factors for VBD [3]. The most sensitive modalities are computed tomography (CT), and magnetic resonance imaging (MRI) and angiography (MRA). Smoker et al. [4] were the first to derive VBD diagnostic criteria; the vertebrobasilar artery is consider to be dilated if its diameter exceeds $4.5 \mathrm{~mm}$, and is considered to be elongated if it lies lateral to the lateral margin of the clivus or dorsum sellae. The MRA standards proposed by Ubogu and Zaidat [5] consider that a basilar artery

Copyright (C) 2020 by Korean Society of Otorhinolaryngology-Head and Neck Surgery.

This is an open-access article distributed under the terms of the Creative Commons Attribution Non-Commercial License (http://creativecommons.org/licenses/by-nc/4.0)

which permits unrestricted non-commercial use, distribution, and reproduction in any medium, provided the original work is properly cited. 
length $>29.5 \mathrm{~mm}$ reflects extension, as does an intracranial, vertebral artery length $>23.5 \mathrm{~mm}$, or a deviation $>10 \mathrm{~mm}$ from the expected course of either artery. Clinical manifestations of VBD vary widely, from asymptomatic status to exertional headache, or even to fatal ischemic stroke or hemorrhage. A mass effect of a tortuous VBD may trigger cranial nerve symptoms attributable to nerve root compression [6]. The facial or trigeminal nerve root is that most commonly affected by adjacent VBD, triggering hemifacial spasm (HFS) or trigeminal neuralgia [7]. Few reports on VBD subjects with vestibulocochlear manifestations have appeared [8]. Such data paucity encouraged us to study VBD subjects with vestibulocochlear symptoms retrospectively. Herein, we review our case series of four VBD subjects presenting with vestibulocochlear symptoms.

\section{MATERIALS AND METHODS}

We retrospectively reviewed the medical records of four VBD subjects with vestibulocochlear symptoms between January 2012 and May 2018. Of these, two were male. We collected demographic information, medical histories, the MRI/MRA findings, and audio-vestibular function test results. This study was approved by the Institutional Review Board at Seoul National University Bundang Hospital (IRB No. B-1810-497-106); the need for informed patient consent was waived.

MRI was performed using a 3T instrument (Achieva and Ingenia; Philips Healthcare, Best, the Netherlands) fitted with a 32-channel Sense head coil (Philips Healthcare). All subjects underwent brain and/or internal auditory canal (IAC) MRI. All MRI protocols included axial T2-weighted images (T2WIs; fieldof-view [FOV], $185 \times 230 \mathrm{~mm}^{2}$; acquisition matrix size, $420 \times$ 375; section thickness, $5 \mathrm{~mm}$; section gap, $1 \mathrm{~mm}$; repetition time [TR], 3,000 ms; echo time [TE], $80 \mathrm{~ms}$; flip angle, $90^{\circ}$ ); and axial gadolinium-enhanced T1-weighted images (T1WIs; FOV, 185× $230 \mathrm{~mm}^{2}$; acquisition matrix size, $420 \times 375$; section thickness, $5 \mathrm{~mm}$; section gap, $1 \mathrm{~mm}$;TR, $560 \mathrm{~ms}$; TE, $10 \mathrm{~ms}$; flip angle, $70^{\circ}$ ) of the whole brain. Three of the four subjects underwent high-resolution T2WI of the IAC featuring three-dimensional (3D), T2-

\section{H I G G H L I G G H T S}

- Vestibulocochlear symptoms can be precipitated by eighth cranial nerve compression by vertebrobasilar dolichoectasia (VBD).

- Appropriate management may improve symptoms in subjects with VBD.

- Radiological evaluation is needed when vestibulocochlear symptoms are not explicable.

- A thorough neurotological evaluation is recommended prior to treatment of VBD. weighted, volume, isotropic, turbo spin-echo acquisition (VISTA) imaging (FOV, $160 \times 160 \mathrm{~mm}^{2}$; acquisition matrix size, $228 \times 228$; section thickness, $0.7 \mathrm{~mm}$; overlap, $0.35 \mathrm{~mm}$; TR, 2,000 ms; TE, $250 \mathrm{~ms}$; flip angle, $90^{\circ}$ ), and 3D gadolinium-enhancing T1WI of the IAC (FOV, $200 \times 200 \mathrm{~mm}^{2}$; acquisition matrix size, $256 \times 256$; section thickness, $1 \mathrm{~mm}$; section gap, $1 \mathrm{~mm}$; TR, $9.5 \mathrm{~ms}$; TE, $3.3 \mathrm{~ms}$; flip angle, $8^{\circ}$ ). Three subjects underwent MRA including time-of-flight intracranial MRA (FOV, $180 \times 180 \mathrm{~mm}^{2}$; acquisition matrix size, $516 \times 359$; section thickness, $1.2 \mathrm{~mm}$; overlap, $0.6 \mathrm{~mm}$; TR, $23 \mathrm{~ms}$; TE, $3.5 \mathrm{~ms}$; flip angle, $18^{\circ}$ ) and gadoliniumenhanced cervical MRA (FOV, $320 \times 350 \mathrm{~mm}^{2}$; acquisition matrix size, $790 \times 550$; section thickness, $1.0 \mathrm{~mm}$; overlap, $0.5 \mathrm{~mm}$; $\mathrm{TR}, 7 \mathrm{~ms}$; TE, $2 \mathrm{~ms}$; flip angle, $27^{\circ}$ ). Two neuroradiologists blinded to clinical findings evaluated the neurovascular structures of the IAC and the cerebellopontine angle, and assessed whether neurovascular contact was evident between the vestibulocochlear nerve and vertebrobasilar artery. Although non-enhanced CT or CT angiography is a sensitive diagnostic option for VBD, MRI was considered in our study for its distinguished function of delineating VBD and adjacent neural structures [9]. The MRI diagnostic criteria for VBD is listed in Table $1[4,5,9,10]$.

Pure tone audiometry (PTA) and speech audiometry tests were performed on three patients reporting subjective hearing loss. The pure-tone average was defined as the average hearing threshold at $0.5,1,2$, and $3 \mathrm{~Hz}$ [11-16]. Sensorineural hearing loss (SNHL) was defined as over $25 \mathrm{~dB}$ of hearing impairment at PTA assessment. Low frequency hearing loss is defined as 70 $\mathrm{dB}$ or more in sum of hearing levels at 125,250 , and $500 \mathrm{~Hz}$, whereas the sum of hearing levels of 2,4 , and $8 \mathrm{kHz}$ are less than $60 \mathrm{~dB}$ or show less than $10 \mathrm{~dB}$ difference of both ears in average hearing level at high frequency $(2,4$, and $8 \mathrm{kHz})$. Two patients presenting with vertigo underwent neurotological examinations. The videonystagmography system (Chartr ENG; ICS Medical, Schaumburg, IL, USA) was used to examine eye movement in the sitting position. Spontaneous nystagmus was analyzed while the subjects attempted to look straight ahead with and without fixation. Positioning- or head-shaking-induced nystagmus was also checked. Head-shaking nystagmus was exam-

Table 1. MRI diagnostic criteria for VBD $[4,5,9,10]$

\begin{tabular}{lcl}
\hline Criterion & Grade & \multicolumn{1}{c}{ MRI } \\
\hline Diameter & & $\leq 4.5 \mathrm{~mm}$ \\
Height of the BA & 0 & At or below the dorsum sellae \\
bifurcation & 1 & Within the suprasellar cistern \\
& 2 & At the level of the third ventricle floor \\
& 3 & Indenting and elevating the third ventricle floor \\
Lateral position & 0 & Midline \\
& 1 & Midline or questionably off midline \\
& 2 & Definitely displaced to the side \\
& 3 & Reaching the cerebellopontine angle \\
\hline
\end{tabular}

$\mathrm{MRI}$, magnetic resonance imaging; VBD, vertebrobasilar dolichoectasia; BA, basilar artery. 
Table 2. Summary of symptoms of the four subjects

\begin{tabular}{|c|c|c|c|c|c|c|c|c|c|c|c|}
\hline Case & Sex & $\begin{array}{l}\text { Age } \\
\text { (yr) }\end{array}$ & $\begin{array}{c}\text { Dolichoectatic } \\
\text { side }\end{array}$ & HFS & $\mathrm{HL}$ & Tinnitus & Vertigo & Duration & Audio & VFT & $\begin{array}{c}\text { Follow-up } \\
\text { (mo) }\end{array}$ \\
\hline 1 & $\mathrm{~F}$ & 70 & $\mathrm{~L}$ & $L^{\text {a) }}$ & L & L (typewriter) & Paroxysmal & $10 \mathrm{yr}$ & LFHL & & 28 \\
\hline 2 & $\mathrm{~F}$ & 71 & $\mathrm{R}$ & - & - & R (typewriter) & Paroxysmala) & 20 day & - & Normal & 2 \\
\hline 3 & M & 68 & $\mathrm{R}$ & $\mathrm{R}$ & $R^{\text {a) }}$ & $\mathrm{R}$ & Recurrent & $6 \mathrm{yr}$ & $\mathrm{SNHL}$ & Caloric weakness: R, 33\% & 24 \\
\hline 4 & M & 37 & L & - & L & $\mathrm{L}^{\text {a) }}$ (tonal) & - & $3 \mathrm{mo}$ & SNHL & - & 7 \\
\hline
\end{tabular}

HFS, hemifacial spasm; HL, hearing loss; VFT, vestibular function test; L, left; R, right; LFHL, low-frequency hearing loss; SNHL, sensorineural hearing loss. a) The chief complaint of each patient.

ined after passive head shaking at a frequency of at least $2 \mathrm{~Hz}$ with the neck flexed by $30^{\circ}$. The head impulse test (HIT) was also performed on one patient with (subjective) moderate-to-severe vertigo. Bithermal caloric tests were performed using a water caloric stimulator (model NCI480, ICS Medical). Irrigation was performed in the order of right cool, left cool, right warm, and left warm. Each irrigation proceeded for 30 seconds at a flow rate of $300 \mathrm{~mL} / \mathrm{min}$ of $\operatorname{cool}\left(30^{\circ} \mathrm{C}\right)$ or warm $\left(44^{\circ} \mathrm{C}\right)$ water. The maximum slow-phase velocity of the nystagmus was determined at each temperature, and canal paresis calculated using the Jongkee's formula.

\section{RESULTS}

As summarized in Table 2, the ipsilesional audiovestibular symptoms of the four VBD patients included SNHL in three, tinnitus in all four, and vertigo in three. Subject 1 of Table 2 presented principally with HFS, but also ipsilateral hearing loss and tinnitus. The principal complaint of subject 2 was vertigo associated with severe tinnitus. The main symptom of subject 3 was lowfrequency hearing loss (LFHL) and tinnitus (that subject had a history of surgery to treat ipsilesional HFS). Subject 4 presented principally with tinnitus and high-frequency hearing loss. Brain MRI/MRA of all four subjects revealed unilateralVBD with neurovascular compression of the cisternal segment or the brainstem causing displacement, angulation, or deformity of cranial nerve VII or VIII.

Subject 1 was a 70 -year-old female with chronic, left-side facial twitching that had commenced 10 years prior. The neurological examination revealed HFS on the left side involving the temporal and marginal mandibular branches of the facial nerve. The symptom was associated with left-sided typewriter tinnitus and paroxysmal vertigo; these developed occasionally along with the HFS. She also presented with fluctuating hearing loss in the left ear. On initial presentation, PTA revealed moderate low-frequency SNHLs of $40 \mathrm{~dB}$ at $250 \mathrm{~Hz}$ and $30 \mathrm{~dB}$ at $500 \mathrm{~Hz}$ on the left side (Fig. 1A). Her MRI/MRA revealed VBD (height of the basilar artery bifurcation, grade 2; lateral position of the basilar artery, grade 1) (Fig. 1B). A prominently dolichoectatic, left, distal vertebral artery compressed the left vestibulocochlear and facial nerves, causing angulation thereof at the root entry/ exit zones and brainstem (Fig. 1B). MRA also revealed a distended, tortuous vertebrobasilar artery (Fig. 1C). Given the aggravating symptoms, microvascular decompression (MVD) surgery was performed. After surgery, the left HFS was relieved immediately but the left-side hearing loss became aggravated (Fig. 1D), probably attributable to surgical trauma. Although paroxysmal vertigo was associated with hearing loss and HFS, vestibular function test is to be evaluated if the symptom aggravates upon the subject's request.

Subject 2, an otherwise healthy 71-year-old female, visited our outpatient clinic complaining of vertigo and right-side typewriter tinnitus that had developed suddenly 20 days prior. Her vertigo was severe (numeric rating scale; 10 out of 10); it occurred 10-20 times a day in a paroxysmal manner, with each episode lasting less than 1 minute. The associated right-sided tinnitus was described as similar to the sound made by a typewriter; the paroxysmal nature of the symptoms was severely disturbing her daily life. Subjectively, the vertigo received a Dizziness Handicap Inventory score of 50. Vestibular function was normal as assessed by the HIT and detailed ocular function testing. Also, facial nerve electroneuronography revealed no abnormal finding. Brain MRI/MRA revealed right-sided VBD with a basilar artery bifurcation height of grade 2 and a basilar artery lateral position of grade 2 . The axial T2-VISTA image revealed neurovascular compression caused by a dolichotic, right, distal vertebral artery, resulting in indentation of the cisternal segments of the right vestibulocochlear and facial nerves (Fig. 2A). Also, tortuosity and lateral displacement of the vertebrobasilar artery were clearly evident on MRA (Fig. 2B). Oxcarbazepine relieved the tinnitus after 6 days and the vertigo after 2 months; the patient was then lost to follow-up.

Subject 3, a 68-year-old male, complained of recent aggravation of right-side hearing loss that had developed suddenly 5 years prior together with recurrent vertigo and ipsilateral tinnitus (Fig. 3A). A follow-up audiogram revealed aggravation of the right-side SNHL (Fig. 3B). A bithermal caloric test conducted at the onset of right-side sudden SNHL (thus 5 years prior) revealed right-side weakness of $33 \%$ (Fig. 3C). He had undergone MVD to treat the right-side HFS 10 years prior, but both the pre- and postoperative audiometry tests had revealed bilaterally normal hearing. MRI/MRA revealed VBD; the basilar artery diameter was $7 \mathrm{~mm}$, the height of the basilar artery bifurcation 

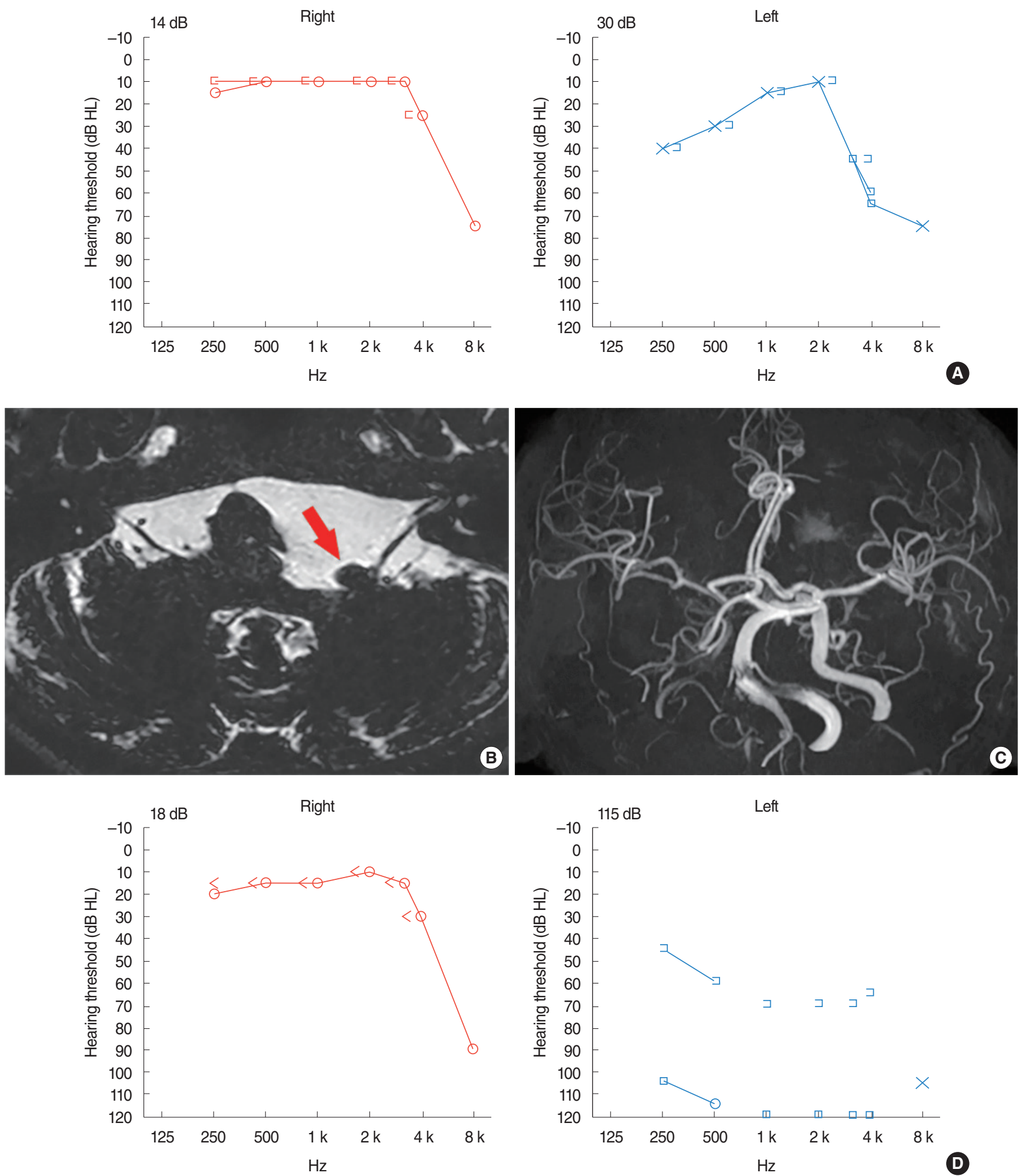

Fig. 1. (A) Pure tone audiometry shows moderate low frequency sensorineural hearing loss on the left side. (B) Axial T2-volume, isotropic, turbo spin-echo acquisition (VISTA) image shows obvious angulation with posterior displacement of the left vestibulocochlear and facial nerves at the root entry/exit zone (arrow) due to neurovascular compression caused by dolichoectatic left distal vertebral artery. Dilated right side vertebrobasilar junction with dark signal intensity is caused by previous coil embolization. (C) Intracranial time-of-flight magnetic resonance angiography well demonstrates vertebrobasilar dolichoectasia with the basilar artery diameter of $4.6 \mathrm{~mm}$, grade 2 height of the basilar artery bifurcation, and grade 1 lateral position of the basilar artery. (D) Pure tone audiometry 2 months after the surgery shows aggravated sensorineural hearing loss on the left side. 

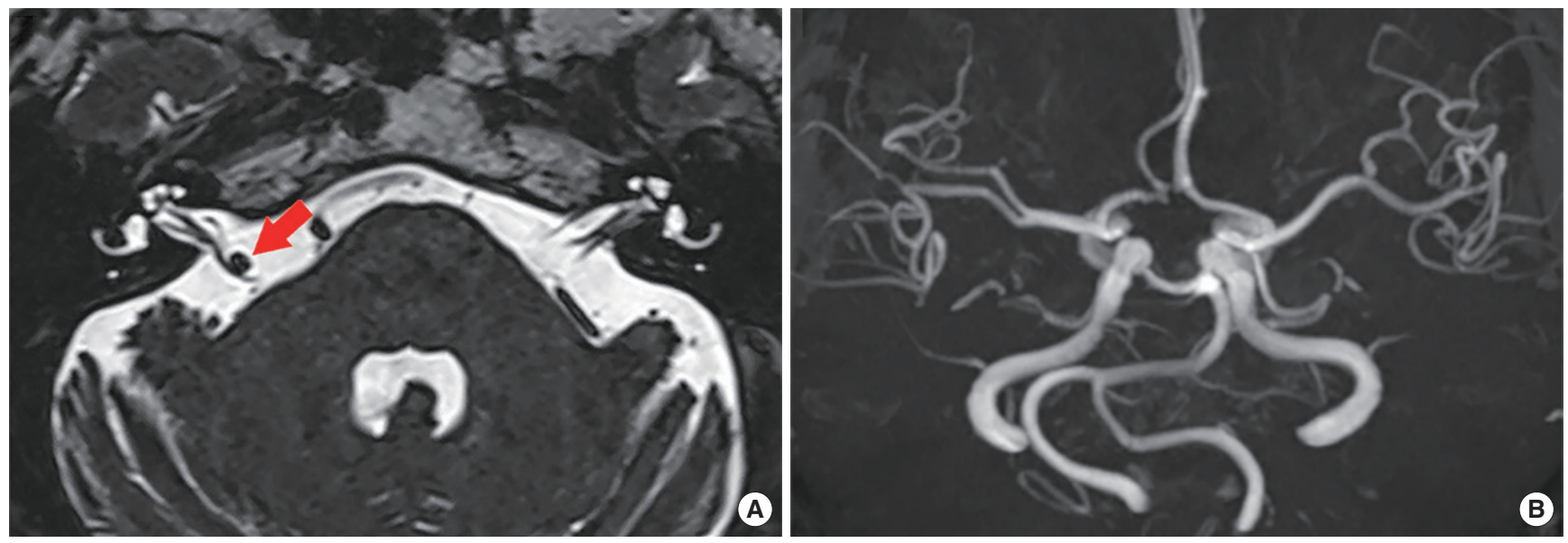

Fig. 2. (A) Axial T2-volume, isotropic, turbo spin-echo acquisition (VISTA) image shows indentation of the right vestibulocochlear and facial nerves at the cisternal segment (arrow) due to neurovascular compression caused by the right distal vertebral artery. (B) Intracranial time-offlight magnetic resonance angiography demonstrates laterally displaced course of the distal vertebral artery. According to Smoker's criteria, the diameter of basilar artery is $3.8 \mathrm{~mm}$, and the grades for the height of the basilar artery bifurcation and lateral position of the basilar artery are all grade 2.
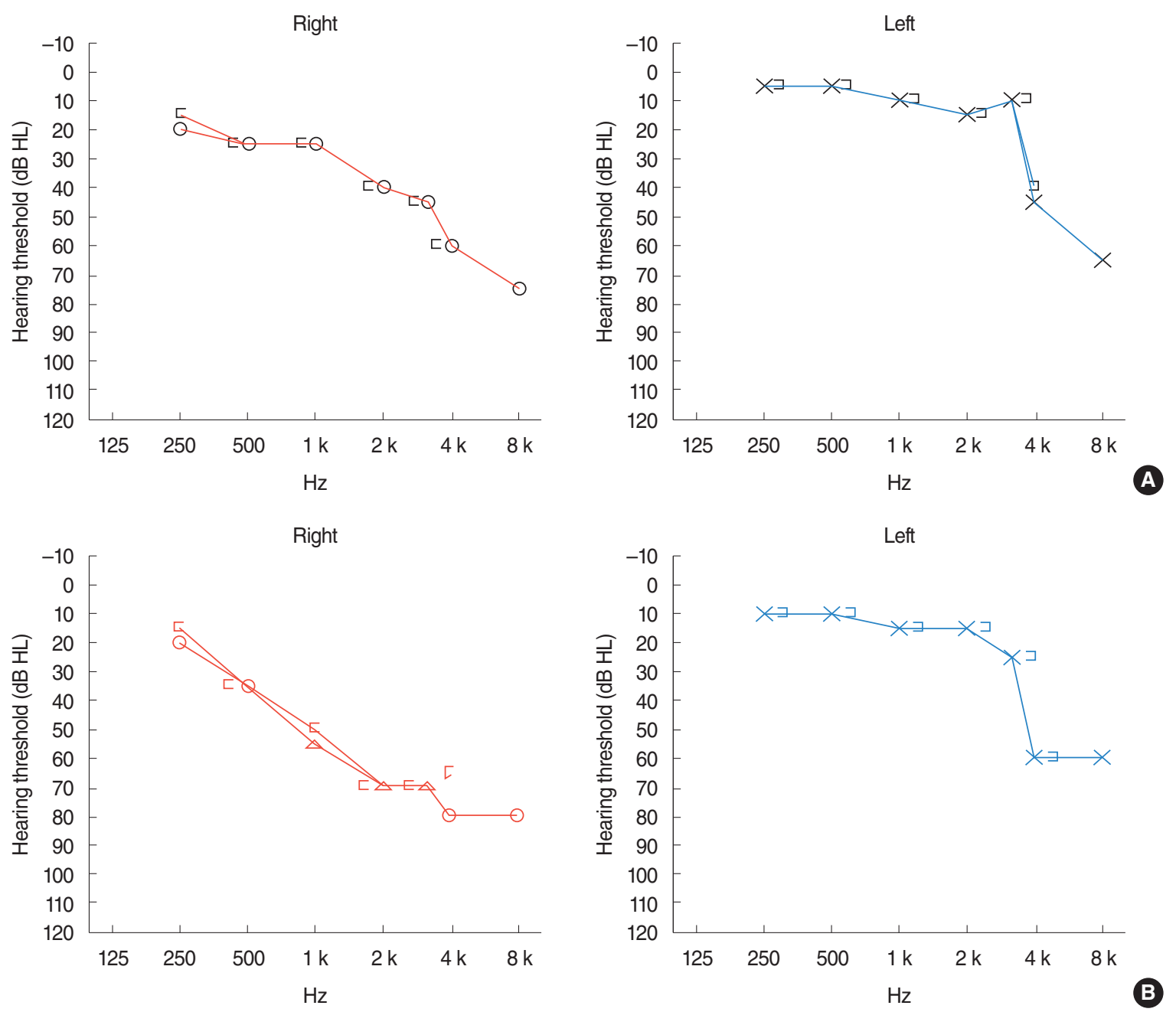

Fig. 3. Pure tone audiometries performed on initial visit $(A)$ and 5 years later $(B)$ reveal aggravating right-sided sensorineural hearing loss. 
Caloric - both eyes

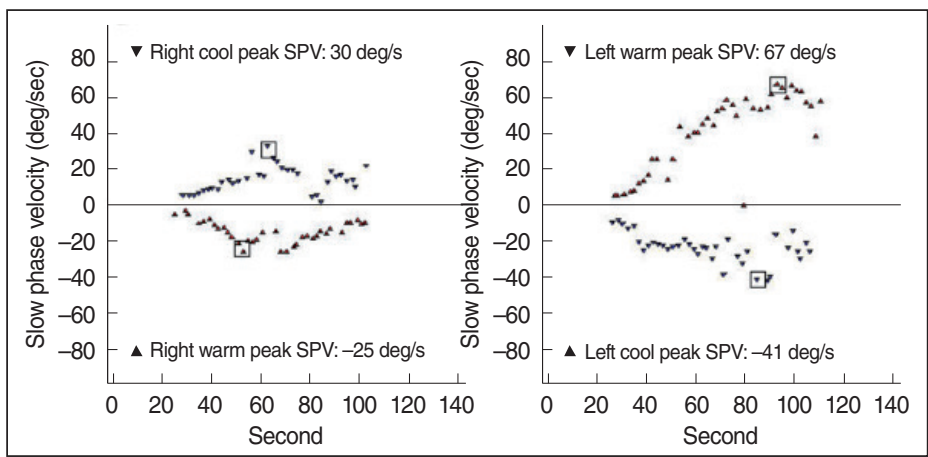

Caloric weakness: $33 \%$ in the right ear

Directional preponderance: $19 \%$ to the left
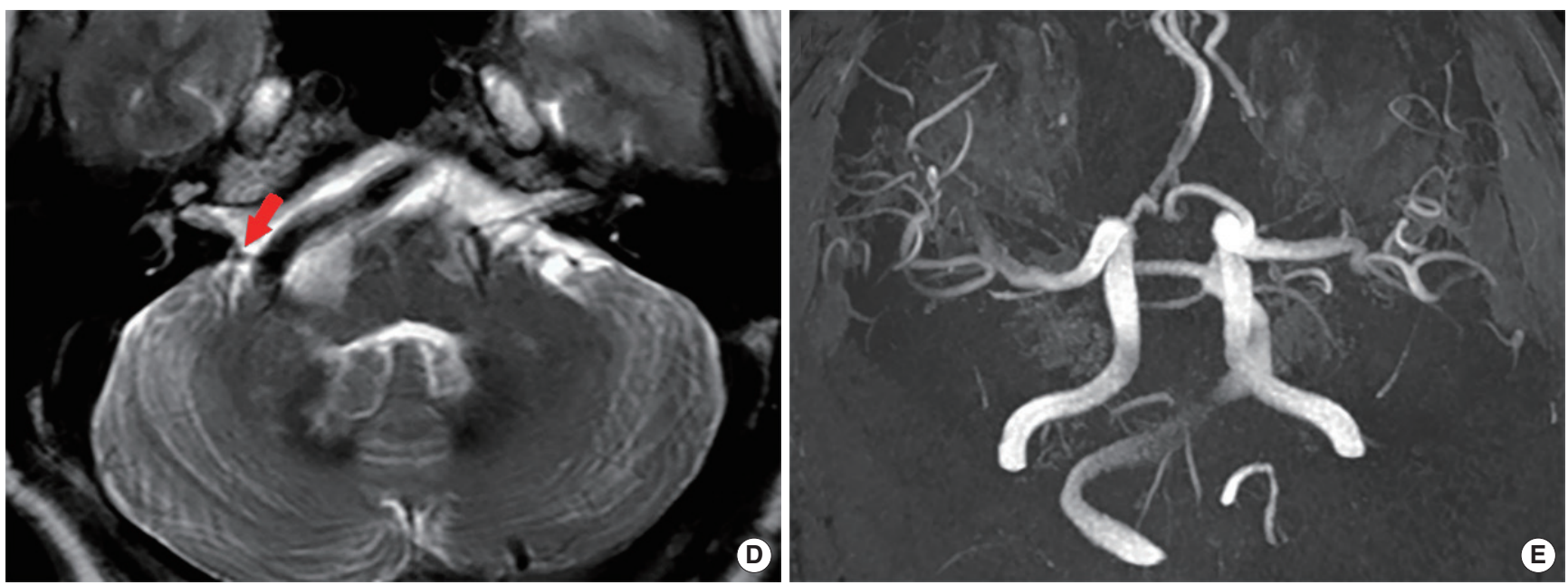

Fig. 3. (Continued) (C) Bithermal caloric test conducted on initial visit showed a 33\% weakness in the right side. (D) Axial T2-weighted images shows the dolichoectatic right distal vertebral artery causes severe posterior displacement of the right vestibulocochlear and facial nerves, probably causing neurovascular compression at the root entry/exit zones and cisternal segments (arrow). (E) Distended and tortuous vertebrobasilar artery is well depicted on the intracranial time-of-flight magnetic resonance angiography. SPV, slow phase velocity.

was of grade 2, and the lateral position of the basilar artery was of grade 3. A dolichoectatic, right, distal, vertebral artery compressed the brainstem along with the right vestibulocochlear and facial nerves, presumably at the root entry/exit zones and the cisternal segments (Fig. 3D and E). The patient refused any treatment and is being followed-up on a regular basis.

Subject 4, a 37-year-old male, presented with bilateral (but left-dominant) tinnitus that had developed 3 months prior. The tinnitus was aggravated upon swallowing and when the neck muscles were tensed. PTA revealed left, unilateral down-sloping SNHL (Fig. 4A). Notably, the auditory brainstem response (ABR) evaluated on suspicion of a retrocochlear etiology revealed significant prolongation of the wave $\mathrm{I}-\mathrm{V}$ interpeak interval of the left compared to the right ear (interaural difference, $0.4 \mathrm{~ms}$ ) (Fig. 4B). The axial T2-VISTA image of IAC-MRI revealed distended, tortuous, left, distal vertebral/anterior, inferior cerebellar arteries triggering neurovascular compression of the left vestibulocochlear and facial nerves at the root entry/exit zones (Fig. 4C and
D), along with compression of the left brainstem. VBD was graded as follows: basilar artery diameter $4.5 \mathrm{~mm}$; basilar artery bifurcation height grade 2 ; and basilar artery lateral position grade 3 . He was conservatively managed using ginkgo biloba extract and tianeptine for 6 months, which was associated with moderate improvement of the tinnitus-related symptoms; he was lost to follow-up thereafter.

\section{DISCUSSION}

VBD is a progressive arterial disease that may directly compress the brainstem and cranial nerves. Clinical manifestation of VBD, often asymptomatic, may vary widely from exertional headache to fatal consequences such as ischemic stroke or hemorrhage. It is considered a potentially severe condition which may cause dysphagia, choking, quadriplegia or hemiparesis due to posterior fossa compression $[5,17]$. Compression of the lower brain- 

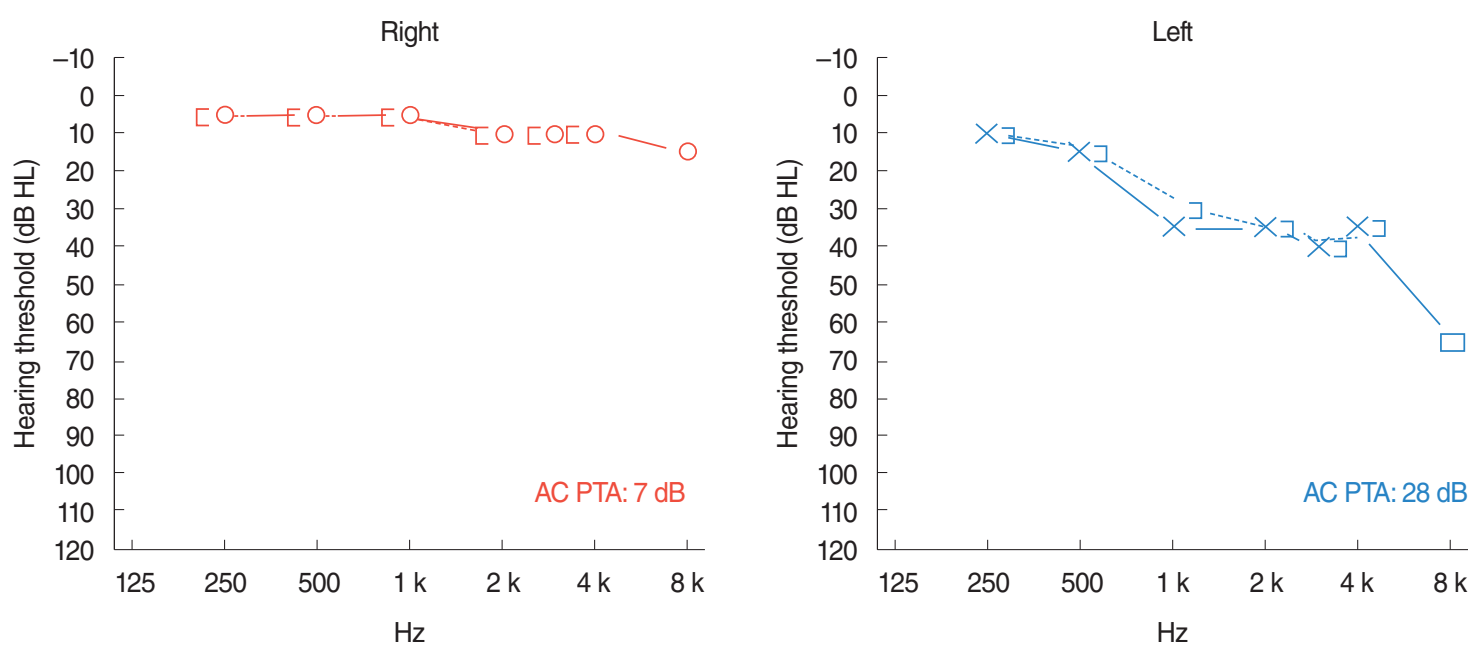

A

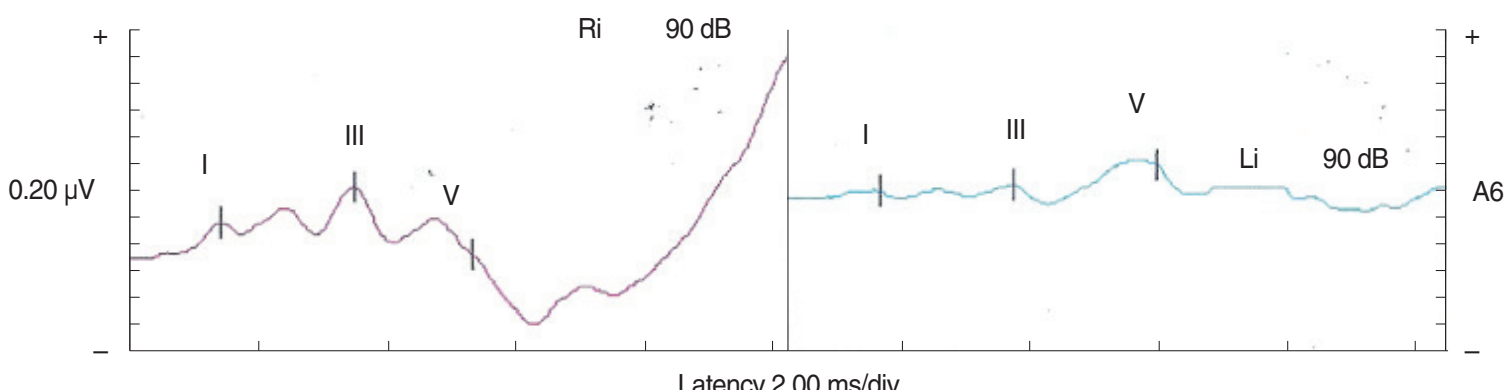

B
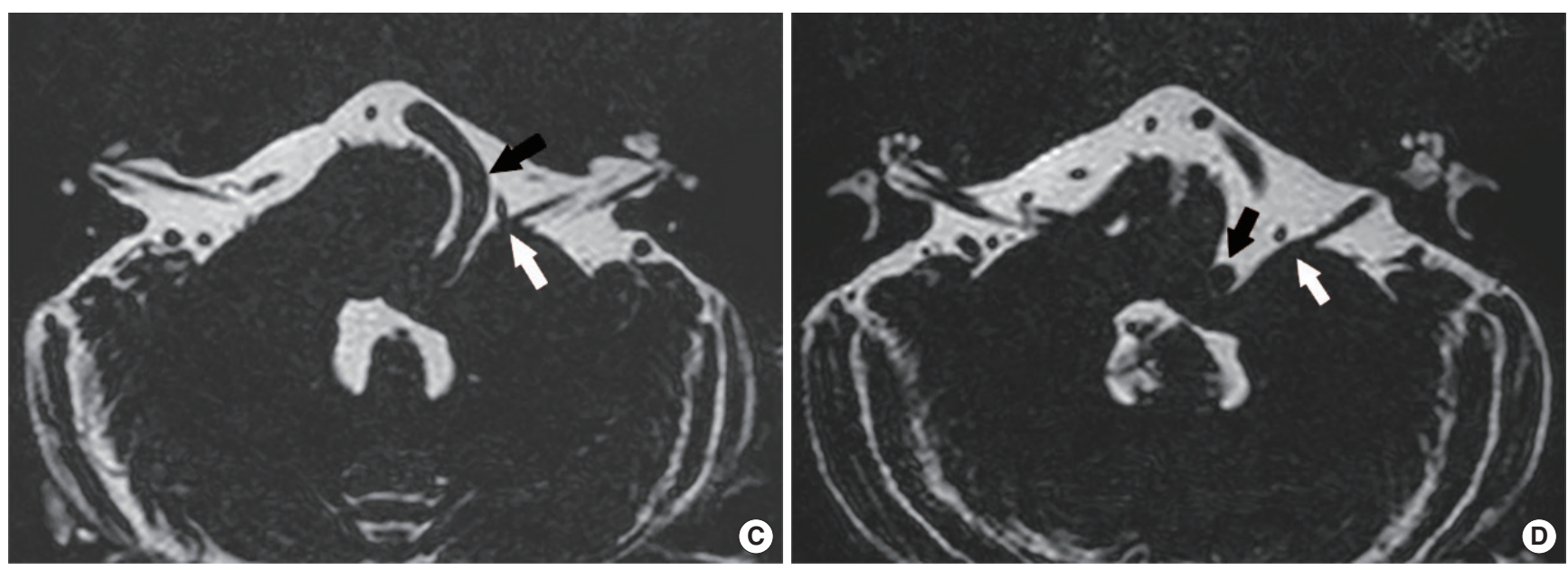

Fig. 4. (A) Pure tone audiometries (PTAs) shows left unilateral down-sloping hearing loss while normal hearing threshold on the right side. (B) Auditory brainstem response shows a significant prolongation of wave I-V interpeak interval of the left ear as compared with the right ear (interaural difference of $0.4 \mathrm{~ms}$ ). (C, D) Axial T2-volume, isotropic, turbo spin-echo acquisition (VISTA) image shows dolichoectatic left distal vertebral artery (black arrows) and left anterior inferior cerebellar artery (white arrows) causing posterior displacement of left vestibulocochlear and facial nerves at the root entry/exit zones. AC, air conduction.

stem can trigger vestibulocochlear symptoms such as hearing loss, tinnitus, and vertigo. Although far fewer reports have dealt with VBD involving the vestibulocochlear nerve than VBD involving the trigeminal or facial nerve, VBD patients commonly present with audiovestibular symptoms [18]. However, the causal relationships between VBD and such symptoms are often difficult to identify because a correspondence between the compression site and the clinical symptoms may not be obvious. The fact that other factors contributing to audiovestibular symptoms are seldom identified renders it even more difficult to confirm a relationship between VBD and such symptoms. Probably because of this, reports on vestibulocochlear symptoms caused by 
VBD are scarce; only a few anecdotal case reports have appeared. For example, a case of unilateral tinnitus caused by VBD [19], a case of downbeat nystagmus probably caused by compressive injury triggered by VBD [20], and a case of unilateral SNHL associated with VBD, were reported recently [8].

Currently, surgery most effectively relieves complications caused by VBD-induced compression injuries. For subjects with HFS, botulinum toxin injection is another treatment option with a relatively low rate of adverse events [21]. Regular toxin injection can be extended to 20 years, but each treatment relieves symptoms for only several months. Therefore, MVD remains the definitive treatment, especially for patients previously treated with botulinum toxin [22]. However, several studies have reported symptom recurrence and serious complications (such as hearing loss and facial nerve palsy) after MVD [23]. Also, MVD may trigger facial hypesthesia, hyperalgesia, and diplopia; in particular, excessive surgical manipulation of vessels can induce cerebellar/brainstem ischemia [24]. Although MVD may thus induce some neurological complications, this surgery remains the most reliable form of treatment as revealed by long-term follow-up studies [25].

In our current case series, subject 1 manifested principally with intolerable HFS associated with ipsilesional low-frequency SNHL, intermittent typewriter tinnitus, and vestibular paroxysmia. The VBD evident on brain MRI/MRA may have compressed not only the facial but also the vestibulocochlear nerve. MVD may have further aggravated the SNHL. As the preoperative SNHL may have been associated with VBD, had an appropriate preoperative neuro-otological examination been conducted, a more focused view of the audiovestibular symptoms may have been possible and postoperative complications anticipated and possibly avoided. The chief complaints of subject 2 , vertigo and tinnitus, were both paroxysmal in nature. The unilateral, staccato-like tinnitus with associated paroxysmal vertiginous spells, and the good response to oxcarbazepine, suggest that typewriter tinnitus and vestibular paroxysmia were in play [26,27]; both are caused by compression of the vestibulocochlear nerve by the anterior-inferior cerebellar artery [28]. However, these complaints of subject 2 were attributable to compression of the vestibulocochlear nerve by VBD. The common characteristics of paroxysmal vertigo in subjects 1 and 2 were diagnosed with vestibular paroxysmia (VP), which is a recurrent spontaneous attack of vertigo lasting for a brief duration (from seconds to minutes). Vertigo attack may or may not be provoked by certain position, and it may be accompanied by unilateral hearing loss or tinnitus. Radiologic confirmation of VBD for these subjects contribute to diagnosis of VP for the neuro-vascular cross-compression demonstrated on MRI falls in the diagnostic criteria of VP. VP could be successfully treated with carbamazepine and oxcarbazepine, whereas surgical treatment could be considered to patients who do not respond to medical treatment [29]. Thus in the case of these two subjects, diagnosis of VBD could aid in planning the treatment, for surgical approach is considerable if symptom persists. Subject 3 had undergone MVD to treat severe HFS 10 years prior.The HFS was relieved and hearing preserved postoperatively, but ipsilesional SNHL developed 5 years later and became gradually aggravated. Although we cannot be sure that the SNHL was attributable only to cochlear nerve compression by VBD, the persistent VBD tortuosity on follow-up MRI/ MRA and the otherwise inexplicable, ipsilesional cochleovestibular loss documented by audio-vestibular function testing support our presumptive diagnosis. In subject 4,VBD compressed the left brainstem rather than the cisternal segment of the vestibulocochlear nerve. Given the ipsilesional nature of the SNHL, prolongation of the wave I-V interpeak interval, and the absence of any other retrocochlear pathology on MRI/MRA, we strongly suspect that the retrocochlear SNHL was caused by compression of the vestibulocochlear nerve by VBD. SNHL in ipsilateral side of VBD were present in three subjects, with the aspect of LFHL for subject 1 and down sloping audiogram for subjects 3 and 4 . The principle complaint for subjects 3 (progressive hearing loss) and 4 (tonal tinnitus) were related to cochlear nerve impairment. The fact that lateral displacement of these two were more severe (grade 3) than other two subjects may imply the clinical correlation of anatomical presentation of VBD and cochlear function, which may be discussed in further studies.

All four subjects presented with symptoms caused by compression of the vestibulocochlear nerve by VBD, but with different principal complaints. Thus, the principal vestibulocochlear symptoms induced by VBD can vary. Whether the audiovestibular symptoms of VBD subjects are caused solely by anatomical compression of the vestibulocochlear nerve, or whether other mechanisms are in play, has previously been debated $[18,20]$. The anterior vestibular artery, which supplies the labyrinth, is particularly vulnerable to ischemia because it lacks collateral vessels [30]. Thus, ischemic injury at a specific location induced by VBD compression, rather than compression per se, may trigger fluctuating, aggravating, or delayed audiovestibular symptoms [18,20,31-33]. Therefore, in subjects with such symptoms, the possibility of vestibulocochlear nerve compression by VBD must be considered and thoroughly evaluated not only via brain or IAC MRI/MRA but also by performing a detailed neurotological examination. Our Subjects 1 and 2 had not been referred to otologists; in addition, they had not received appropriate pretreatment or audiovestibular evaluations. Subjects 2 and 4 were lost to follow up after short follow-up period during conservative management. Although the symptoms improved after treatment, the progress of VBD and associated symptoms are currently not under surveillance, and this may be a weak point of our case series. Although the prevalence of audiovestibular symptoms induced by VBD may be low, a precise diagnosis aids treatment selection and predicts the clinical course. Also, if VBD is confirmed radiologically, a meticulous neurotological evaluation including audiometry, $\mathrm{ABR}$, and detailed vestibular function testing should be performed before initial treatment. Depending 
on the principal complaints and the extent to which VBD compresses the vestibulocochlear nerve, MVD, or carbamazepineor gabapentin-based medication, may be considered.

Taken together, vestibulocochlear symptoms such as SNHL, paroxysmal vertigo, or typewriter tinnitus can be precipitated by neurovascular compression of the eighth cranial nerve by VBD. As appropriate management including MVD, or carbamazepine- or gabapentin-based medication, may stop disease progression and/or improve symptoms in subjects with VBD, a high index of suspicion and meticulous radiological evaluation are needed when vestibulocochlear symptoms are not otherwise explicable. Moreover, if VBD is confirmed and is considered to cause audiovestibular manifestations, a thorough neurotological evaluation is recommended prior to treatment.

\section{CONFLICT OF INTEREST}

No potential conflict of interest relevant to this article was reported.

\section{ACKNOWLEDGMENTS}

This work was supported by a grant from the National Research Foundation of Korea (NRF) grant funded by the Korea government (MSIP) (grant No. NRF-2019R1A2C2004941).

\section{ORCID}

$\begin{array}{ll}\text { Gene Huh } & \text { https://orcid.org/0000-0003-4487-1361 } \\ \text { Yun Jung Bae } & \text { https://orcid.org/0000-0002-1779-4949 } \\ \text { Hyun Jun Woo } & \text { https://orcid.org/0000-0002-7822-2151 } \\ \text { Jung Hyun Park } & \text { https://orcid.org/0000-0001-8636-569X } \\ \text { Ja-Won Koo } & \text { https://orcid.org/0000-0002-5538-2785 } \\ \text { Jae-Jin Song } & \text { https://orcid.org/0000-0002-6631-3232 }\end{array}$

\section{AUTHOR CONTRIBUTIONS}

Conceptualization: YJB. JWK, JJS. Data curation: GH, YJB, HJW, JHP. Formal analysis: GH, YJB. Funding acquisition: JJS. Methodology, Project administration, \& Visualization: GH, JJS. Writing - original draft: GH, YJB, JJS. Writing - review \& editing: all authors.

\section{REFERENCES}

1. Wolters FJ, Rinkel GJ,Vergouwen MD. Clinical course and treatment of vertebrobasilar dolichoectasia: a systematic review of the literature. Neurol Res. 2013 Mar;35(2):131-7.
2. Lou M, Caplan LR. Vertebrobasilar dilatative arteriopathy (dolichoectasia). Ann NY Acad Sci. 2010 Jan;1184(1):121-33.

3. Ikeda K, Nakamura Y, Hirayama T, Sekine T, Nagata R, Kano O, et al. Cardiovascular risk and neuroradiological profiles in asymptomatic vertebrobasilar dolichoectasia. Cerebrovasc Dis. 2010 Jun;30(1): 23-8.

4. SmokerWR, Price MJ, KeyesWD, Corbett JJ, Gentry LR. High-resolution computed tomography of the basilar artery: 1 . normal size and position. AJNR Am J Neuroradiol. 1986 Jan-Feb;7(1):55-60.

5. Ubogu EE, Zaidat OO. Vertebrobasilar dolichoectasia diagnosed by magnetic resonance angiography and risk of stroke and death: a cohort study. J Neurol Neurosurg Psychiatry. 2004 Jan;75(1):22-6.

6. Passero SG, Rossi S. Natural history of vertebrobasilar dolichoectasia. Neurology. 2008 Jan;70(1):66-72.

7. El-Ghandour NM. Microvascular decompression in the treatment of trigeminal neuralgia caused by vertebrobasilar ectasia. Neurosurgery. 2010 Aug;67(2):330-7.

8. Gilbow RC, Ruhl DS, Hashisaki GT. Unilateral sensorineural hearing loss associated with vertebrobasilar dolichoectasia. Otol Neurotol. 2018 Jan;39(1):e56-7.

9. Samim M, Goldstein A, Schindler J, Johnson MH. Multimodality imaging of vertebrobasilar dolichoectasia: clinical presentations and imaging spectrum. Radiographics. 2016 Jul-Aug;36(4):1129-46.

10. SmokerWR, Corbett JJ, Gentry LR, KeyesWD, Price MJ, McKusker S. High-resolution computed tomography of the basilar artery: 2 . vertebrobasilar dolichoectasia: clinical-pathologic correlation and review. AJNR Am J Neuroradiol. 1986 Jan-Feb;7(1):61-72.

11. Lee SY, Nam DW, Koo JW, De Ridder D, Vanneste S, Song JJ. No auditory experience, no tinnitus: lessons from subjects with congenital- and acquired single-sided deafness. Hear Res. 2017 Oct;354:9-15.

12. Jeon HW, Kim SY, Choi BS, Bae YJ, Koo JW, Song JJ. Pseudo-low frequency hearing loss and its improvement after treatment may be objective signs of significant vascular pathology in patients with pulsatile tinnitus. Otol Neurotol. 2016 Oct;37(9):1344-9.

13. Kim CS, Kim SY, Choi H, Koo JW,Yoo SY,An GS, et al.Transmastoid reshaping of the sigmoid sinus: preliminary study of a novel surgical method to quiet pulsatile tinnitus of an unrecognized vascular origin. J Neurosurg. 2016 Aug;125(2):441-9.

14. Song JJ, Hong SK, Lee SY, Park SJ, Kang SI, An YH, et al.Vestibular manifestations in subjects with enlarged vestibular aqueduct. Otol Neurotol. 2018 Jul;39(6):e461-7.

15. Bae YJ, Song JJ, Choi BS, Kang Y, Kim JH, Koo JW. Differentiation between intralabyrinthine schwannoma and contrast-enhancing labyrinthitis on MRI: quantitative analysis of signal intensity characteristics. Otol Neurotol. 2018 Sep;39(8):1045-52.

16. Shim YJ, Bae YJ, An GS, Lee K, Kim Y, Lee SY, et al. Involvement of the internal auditory canal in subjects with cochlear otosclerosis: a less acknowledged third window that affects surgical outcome. Otol Neurotol. 2019 Mar;40(3):e186-90.

17. He X, Duan C, Zhang J, Li X, Zhang X, Li Z.The safety and efficacy of using large woven stents to treat vertebrobasilar dolichoectasia. J Neurointerv Surg. 2019 Jun 13 [Epub]. https://doi.org/10.1136/ neurintsurg-2019-014933.

18. Passero S, Nuti D. Auditory and vestibular system findings in patients with vertebrobasilar dolichoectasia. Acta Neurol Scand. 1996 Jan; 93(1):50-5.

19. Titlic M,Tonkic A, Jukic I, Buca A, Kolic K, Batinic T.Tinnitus caused by vertebrobasilar dolichoectasia. Bratisl Lek Listy. 2007;108(10-11): 455-7.

20. Jacobson DM, Corbett JJ. Downbeat nystagmus and dolichoectasia of the vertebrobasilar artery. J Neuroophthalmol. 2002 Jun;22(2): 150-1.

21. Czyz CN, Burns JA, PetrieTP,Watkins JR, Cahill KV, Foster JA. Longterm botulinum toxin treatment of benign essential blepharospasm, 
hemifacial spasm, and Meige syndrome. Am J Ophthalmol. $2013 \mathrm{Jul}$; 156(1):173-7.

22. Wang X, Thirumala PD, Shah A, Gardner P, Habeych M, Crammond DJ, et al. Effect of previous botulinum neurotoxin treatment on microvascular decompression for hemifacial spasm. Neurosurg Focus. 2013 Mar;34(3):E3.

23. Savitz SI, Ronthal M, Caplan LR. Vertebral artery compression of the medulla.Arch Neurol. 2006 Feb;63(2):234-41.

24. Sun S, Jiang W, Wang J, Gao P, Zhang X, Jiao L, et al. Clinical analysis and surgical treatment of trigeminal neuralgia caused by vertebrobasilar dolichoectasia: a retrospective study. Int J Surg. 2017 May; 41:183-9.

25. Barker FG 2nd, Jannetta PJ, Bissonette DJ, Larkins MV, Jho HD.The long-term outcome of microvascular decompression for trigeminal neuralgia. N Engl J Med. 1996Apr;334(17):1077-83.

26. Sunwoo W, Jeon YJ, Bae YJ, Jang JH, Koo JW, Song JJ. Typewriter tinnitus revisited: the typical symptoms and the initial response to carbamazepine are the most reliable diagnostic clues. Sci Rep. 2017 Sep;7(1):10615.

27. Bae YJ, Jeon YJ, Choi BS, Koo JW, Song JJ. The role of MRI in diagnosing neurovascular compression of the cochlear nerve resulting in typewriter tinnitus. AJNR Am J Neuroradiol. 2017 Jun;38(6):1212-7.

28. Mardini MK. Ear-clicking "tinnitus" responding to carbamazepine. N Engl J Med. 1987 Dec;317(24):1542.

29. Hufner K, Barresi D, Glaser M, Linn J,Adrion C, Mansmann U, et al. Vestibular paroxysmia: diagnostic features and medical treatment. Neurology. 2008 Sep;71(13):1006-14.

30. Mazzoni A. Internal auditory artery supply to the petrous bone.Ann Otol Rhinol Laryngol. 1972 Feb;81(1):13-21.

31. Han SA, Choe G, Kim Y, Koo JW, Choi BY, Song JJ. Beware of a fragile footplate: lessons from ossiculoplasty in patients with ossicular anomalies related to second pharyngeal arch defects. J Clin Med. 2019 Dec;8(12):2130.

32. Bae YJ, Shim YJ, Choi BS, Kim JH, Koo JW, Song JJ. "Third window" and "single window" effects impede surgical success: analysis of retrofenestral otosclerosis involving the internal auditory canal or round window. J Clin Med. 2019 Aug;8(8):1182.

33. Han J, De Ridder D, Vanneste S, Chen YC, Koo JW, Song JJ. Pre-treatment ongoing cortical oscillatory activity predicts improvement of tinnitus after partial peripheral reafferentation with hearing aids. Front Neurosci. 2020;14:410. 